\title{
Expression of Tight Junction Proteins Is Altered in Bladder Cancer
}

\author{
Bangbin Chen $(\mathbb{D}$, Renge Bu $\mathbb{D}$, and Xuewen $\mathrm{Xu} \oplus$ \\ Department of Urology, Shengjing Hospital of China Medical University, Shenyang, Liaoning, China 110004 \\ Correspondence should be addressed to Xuewen Xu; xuxw@sj-hospital.org
}

Received 9 June 2020; Accepted 3 November 2020; Published 17 November 2020

Academic Editor: Lubna H. Tahtamouni

Copyright ( $) 2020$ Bangbin Chen et al. This is an open access article distributed under the Creative Commons Attribution License, which permits unrestricted use, distribution, and reproduction in any medium, provided the original work is properly cited.

\begin{abstract}
Bladder cancer (BC) is one of the tumors which occur most frequently in urological system, but less is known about the expression of tight junction proteins and its clinical significance in BC. In this study, expression of claudin-4, zonula occludens-1 (ZO-1) and zonula occludens-1 nucleic acid-binding protein (ZONAB), in BC tissues, adjacent nontumor tissue (ANTT), and BC cell lines was examined by Western blotting, semiquantitative RT-PCR, and immunohistochemistry, and then, the clinical significance of these proteins was investigated. The mRNA and protein expression of ZONAB were significantly upregulated, while those of ZO-1 was significantly downregulated in some BC cell lines and tissues in comparison with nontumor urothelial cell lines and ANTT. High expression rate of ZO-1 and ZONAB had negative correlation in $\mathrm{BC}$ tissues and was also correlated with muscle-invasive lesions in $\mathrm{BC}$ tissues. In conclusion, the expression of tight junction proteins is significantly altered in $\mathrm{BC}$ and $\mathrm{ZO}-1$, and ZONAB interaction might be involved in $\mathrm{BC}$ development.
\end{abstract}

\section{Introduction}

Bladder cancer (BC) is one of the tumors which occur most frequently in the urological system. Although there are more and more treatments for $\mathrm{BC}$, the recurrence rate and mortality rate are still relatively high, which makes it more urgent to explore the mechanism of tumorigenesis [1]. Intercellular adhesion is the first barrier against development of solid tumors [2]. As an important intercellular junction, tight junction turns out to be important in inhibition of neoplastic proliferation and metastasis $[3,4]$. Tight junction is a protein strand that seals the gap between adjacent cells and separates the lateral intercellular space from apical lumen of the epithelium [5]. It also participates in intercellular signaling and involves in several biological processes [6-8]. Tight junctions consist of three groups of proteins: integral membrane proteins, plaque anchoring proteins, and tight junction regulatory proteins $[9$, 10]. The extracellular domain of the membrane protein binds to corresponding tight junction protein of adjacent cells [11, 12]. The intracellular domain binds to the plaque anchoring protein which is located on the cell membrane's inner surface and binds to the cytoskeletal protein [13]. The integrity of tight junctional component is vital for the maintenance of cell polarity, morphology, and barrier function $[10,14,15]$.
Altered expression of tight junction proteins attenuates the barrier function against cancer development $[2,16,17]$. In order to define the antitumor function of tight junction, it is necessary to detect the expression level of those proteins in malignant tumors [18]. Claudin-4 is one important integral membrane protein belonging to tight junction. Claudin- 4 expression is altered in several solid tumors. For example, its expression in triple-negative breast cancer is markedly upregulated and downregulated in colorectal cancer $[19,20]$. Overexpression of that in ovarian cancer predicts a poor 5-year survival rate [21]. ZO-1 is one of plaque anchoring proteins of tight junction and its abnormal expression is also observed in some malignancies. ZO-1 expression is significantly decreased in non-small cell lung cancer [22]. It is also involved in ZIP4 initiated pancreatic cancer invasion [23]. ZONAB as a tight junction regulatory protein is involved in estrogen-induced breast cancer metastasis [24]. ZONAB is also involved in the development of ethanolinduced gastric cancer [25]. Claudin-4, ZO-1, and ZONAB serve as important proteins of tight junction, and their normal expression is vital for the barrier function, particularly the process of cancer cell proliferation as well as metastasis. However, the expression profile of these proteins in $\mathrm{BC}$ has not been systematically examined. 
Based on the findings of claudin-4, ZO-1, and ZONAB expression in other solid tumors, we speculate that their expression in $\mathrm{BC}$ is also altered. We expect to have further understanding on the integrity of tight junction structure and its correlation with clinical manifestation in BC. By doing so, we provide a basis for defining the specific character of tight junction component in the development of BC. In order to verify our hypothesis, we examined $\mathrm{BC}$ tissues and ANTT of their claudin-4, ZO-1, and ZONAB expression and analyzed their correlation with each other as well as clinicopathological factors.

\section{Materials and Methods}

2.1. Tissue Samples. 53 paired BC and ANTT specimens were obtained from patients undergoing surgery between January 2016 and January 2017 at the Department of Urology, Shengjing Hospital Affiliated to China Medical University, Shenyang, China. All patients were diagnosed by pathology with bladder urothelial carcinoma. BC tissues and ANTT (tumor-free bladder tissues taken $2 \mathrm{~cm}$ away from the edge of the tumor) specimens were obtained and washed with RNase-free $0.9 \%$ sodium chloride to eliminate blood intraoperatively within 5 minutes after resection. Nontumor tissues were removed from tumor specimens, and smooth muscle from ANTT. Partial samples for RNA extraction were preserved, stored in liquid nitrogen at once, and then transferred to a $-80^{\circ} \mathrm{C}$ deep-freeze. Histological examination confirmed that more than $80 \%$ of the frozen $\mathrm{BC}$ tissue consisted of tumor cells for RNA extraction. The remaining sample was fixed in $10 \%$ formalin for pathological examination and immunohistochemical staining.

2.2. Cell Lines. The human noncancer urothelial cell line svhuc- 1 and bladder urothelial carcinoma cell lines 5637, UM-UC-3, and T24 were obtained through the Cell Bank of Type Culture Collection of the Chinese Academy of Sciences (Shanghai). We got sv-huc-1 cells cultured in F12K, UM-UC-3 cells in DMEM, T24, and 5637 cells in RPMI 1640 medium all with $10 \%$ heat-inactivated fetal bovine serum (FBS) at $37^{\circ} \mathrm{C}$ under $5 \% \mathrm{CO} 2$.

2.3. Western Blotting. RIPA cell lysate in suitable volume was added to the cells. After centrifugation, by the bicinchoninic acid (BCA) protein concentration assay kit (Beyotime, Shanghai, China), we got the concentration of the protein in the supernate. Before being diluted by a $5 \times$ Loading Buffer, the samples were boiled with water bath lasting for 5 minutes. Then, we used SDS-PAGE to separate the protein and transferred them to a PVDF membrane (Millipore, USA). Since being incubated with primary antibody, including mouse anti- $\beta$-actin monoclonal antibody (diluted $1: 1000$; Cell Signaling Technology), mouse anti-claudin-4 monoclonal antibody (diluted 1:500; Invitrogen), rabbit anti-ZO-1 polyclonal antibody (diluted $1: 250$; Invitrogen), rabbit antiZONAB polyclonal antibody (diluted 1:1000; Invitrogen), and goat secondary antibody conjugated to horseradish peroxidase (diluted 1:5000; Sangon, Shanghai, China), ECL luminescence solution (Sigma, St. Louis, MO) was added as luminous substrate, and the band intensity was analyzed by a digital scanning imaging system.

2.4. RNA Expression Analysis. For the detection of RNA expression, we used the method of semiquantitative RTqPCR. We washed the collected cell samples twice through PBS, froze tissue samples in liquid nitrogen, and then ground them to powder. By adding TRIzol reagent, we could extract the total RNA. The extracted RNA absorbance was measured using a spectrophotometer to calculate concentration. By reverse transcription through the PrimeScript RT Master Mix kit (Takara Biotechnology Co., Ltd., Japan), total RNA was used to develop cDNA. Real-time PCR was conducted with cDNA as the template using the SYBR Premix Ex Taq II kit (Takara Biotechnology Co., Ltd., Japan) within the AriaMx real-time fluorescence PCR system (Agilent, USA). PCR amplification conditions were as follows: $95^{\circ} \mathrm{C}$ denaturation $30 \mathrm{~s}, 45 \mathrm{PCR}$ cycles $\left(95^{\circ} \mathrm{C} 5 \mathrm{~s} ; 60^{\circ} \mathrm{C} 20 \mathrm{~s}\right), 65^{\circ} \mathrm{C}$ extended $10 \mathrm{~s}$, and $65^{\circ} \mathrm{C}$ fluorescence signal detection, to obtain the standard curve. We took GAPDH mRNA as internal control and water as a negative control. Primer sequences turned out to be as follows: claudin-4 upstream: $5^{\prime}$-AGCTCTGTGGC CTCAGGACTCT-3', downstream: $5^{\prime}$-CAGTGATGAAT AGCTCTTCTTAAATTACAA-3'; ZO-1 upstream: $5^{\prime}$ -GGATGTTTATCGTCGCATTGTA-3', downstream: $5^{\prime}$ -AAGAGCCCAGTTTTCCATTGTA-3' ; ZONAB upstream: 5'-GCTGGGGAGGAGGAGGA-3' , downstream: $5^{\prime}$-CTGT TGGGATGGGGTAAGAC-3' ; and GAPDH upstream: $5^{\prime}$ -TCCCTGAGCTGAACGGGAAG-3', downstream: $5^{\prime}$ -GGAGGAGTGGGTGTCGCTGT-3'. The relative expression level of the each gene was obtained according to the standard curve. Statistical analysis was performed using the $2^{-\Delta \Delta \mathrm{Cq}}$ method.

2.5. Immunohistochemistry. Before putting BC tissues and ANTT in sodium citrate in microwave for antigen retrieval, we fixed them in formalin, embedded them in paraffin, sectioned them at $5 \mu \mathrm{m}$ thick, and then dewaxed and rehydrated them. The slides were added by a nonspecific stain blocking agent, and then incubated them at room temperature for 30 minutes. Anti-human claudin-4 primary antibody $(1: 100$; Zymed, San Francisco, CA, USA) or anti-human ZO-1 primary antibody (1:300, Abcam, Cambridge, MA) or antihuman ZONAB primary antibody (1: 200, Thermo Fisher, Shanghai, China) was added and incubated for a whole night at $4^{\circ} \mathrm{C}$. We took PBS for slide washing, and then incubated them with secondary antibody $(1: 100$, Gene Tech, Shanghai, China) for 30 minutes. After washing off excessive secondary antibody, we incubated them with Streptavidin-ABC, got them developed by diaminobenzaminidine (DAB), washed, counterstained, dehydrated, dried, and finally mounted by neutral balsam. Under the microscope, we observed and photographed tissue morphology and compared them with sections which were already known positive. Two pathologists were invited to evaluate the results blindly. For each section, 5 high power fields (original magnification $\times 400$ ) were selected at random, and in every field, 200 cells were observed. We acquire semiquantitative results by confirming 


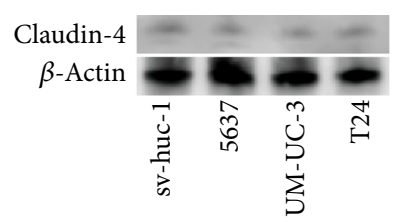

Claudin-4

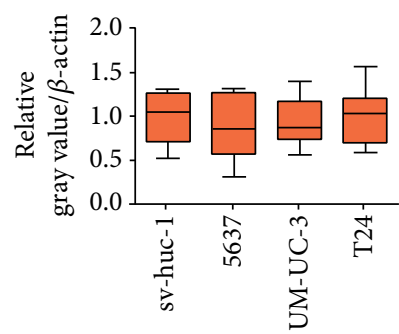

(a)

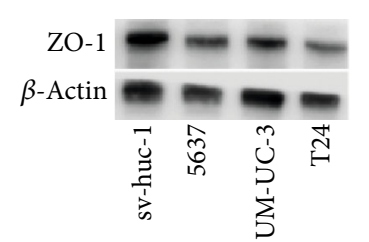

ZO-1

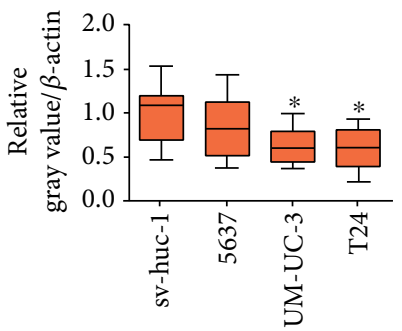

(b)

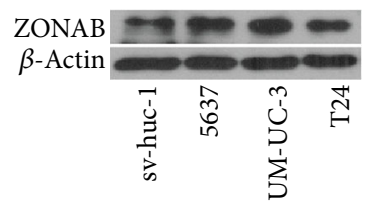

ZONAB

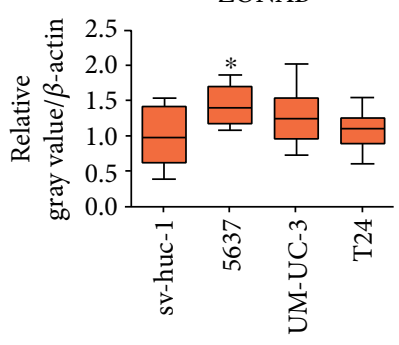

(c)

FIGURE 1: ZO-1 expression is downregulated and ZONAB expression is upregulated in some bladder cancer cell lines. The expression of claudin-4 (a), ZO-1 (b), and ZONAB (c) protein in noncancer cell line (sv-huc-1) and bladder cancer cell lines (5637, UM-UC-3, and T24) was detected by Western blot. The relative grey value adjusted to the internal control $\beta$-actin and then standardized to the value of sv-huc-1 is shown by box and whisker plots. The whiskers represent the minimal or the maximal gray value, and the boxes span the interquartile range of measurements for cell lines with the mean value of 10 replicates $(n=10) . * P<0.05$, one-way ANOVA, post hoc Tukey's test.

staining intensity and scale. Positive cells were defined as follows: any cell whose nucleus or cytoplasm were stained dark brown or yellow. Cell staining intensity score: 0 for no staining, 1 for weak staining, 2 for moderate staining, and 3 for strong staining. The cell staining scale score was as follows: 0 for positive cells $\leq 5 \%, 1$ for $6-25 \%, 2$ for $6-50 \%, 3$ for 51 $75 \%, 4$ for $>75 \%$. The composite staining score (cell staining intensity score + cell staining scale score) is as follows: 2 points were considered negative staining (low staining), 3 to 4 points were considered moderate staining, and 5 to 6 points were considered high staining.

2.6. Statistical Analysis. Data analysis was conducted using a statistical program by the GraphPad Prism 7 software, plotting box, and whisker graphs as well as stacked bars. We conducted three independent and repeated trials, acquired results of each assay, then had them expressed as whiskers (the minimal and the maximal value) as well as the boxes (the interquartile range of the measure). For comparing those numerical data from the two groups, we performed Student's $t$-test. One-way ANOVA together with post hoc Tukey's multiple comparison test was conducted as well for comparing the statistical difference between more than two groups. As for categorical data, Fisher's exact test could show their difference. Correlation analysis of the data was processed using Spearman's rank order correlation coefficient.

\section{Results}

The tight junction protein expression is often changed in solid tumors [19-25]. To identify their expression pattern in $\mathrm{BC}$, we detected claudin-4, $\mathrm{ZO}-1$, and $\mathrm{ZONAB}$ protein level in 1 human noncancer urothelial cell line sv-huc-1 with 3 human BC cell lines 5637, UM-UC-3, and T24 through Western blotting. The findings showed the tight junction protein expression within $\mathrm{BC}$ cells is altered. The mRNA 

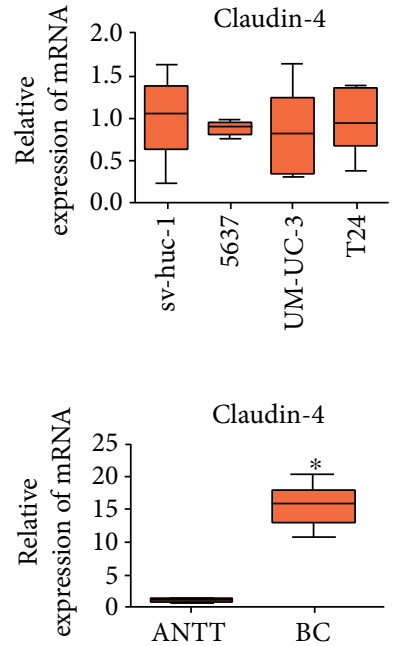

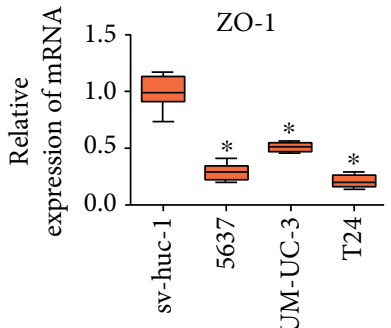

(a)

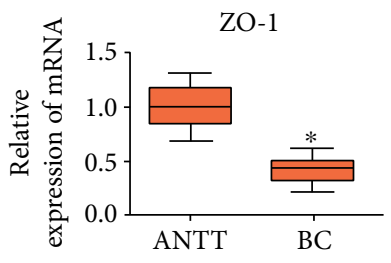

(b)
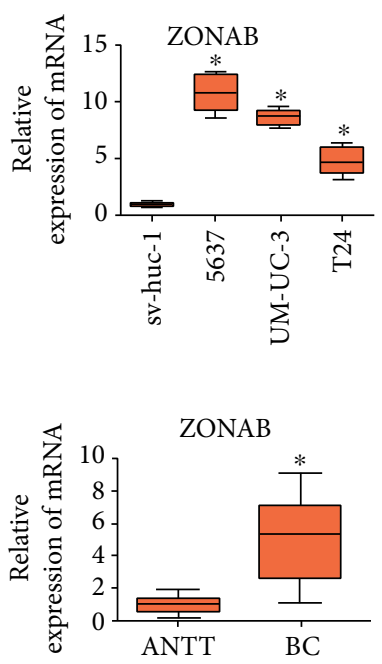

FIgURE 2: The expression of ZO-1 mRNA is downregulated and ZONAB mRNA is upregulated in bladder cancer (BC) cell lines and BC tissues. (a) The expression of claudin-4, ZO-1, and ZONAB mRNA in noncancer cell line (sv-huc-1) and bladder cancer cell lines (5637, UM-UC-3, and T24) was detected by real-time PCR. Relative expression adjusted to the reference gene GAPDH and then standardized to sv-huc-1 is shown by box and whisker plots. The whiskers represent the minimal or the maximal value and the boxes spans the interquartile range of measurements for cell lines with the mean value of 10 replicates $(n=10) . * P<0.05$, one-way ANOVA, post hoc Tukey's test. (b) The expression of claudin-4, ZO-1, and ZONAB mRNA in BC tissue and adjacent nontumor tissues (ANTT) was detected by real-time PCR. Relative expression adjusted to the reference gene GAPDH and then standardized to the value of ANTT is shown by box and whisker plots. The whiskers represent the minimal or the maximal value, and the boxes span the interquartile range of measurements for 53 patients with the mean value of 3 replicates $(n=53) * P<0.05$, Student's $t$-test.

expression of tight junction proteins is also altered both in BC cell lines and tissues. Claudin-4, ZO-1, and ZONAB mRNA expression was detected in sv-huc-1, 5637, UMUC-3, and T24 cell lines through real-time PCR to verify those findings from Western blotting. The results of RNA expression analysis were consistent with the observations in the protein expression analysis.

To validate the result in these cell lines, we detected claudin-4, ZO-1, and ZONAB mRNA level in 53 paired ANTT and $\mathrm{BC}$ tissue samples. Data were gathered form 42 males and 11 females with a mean age of 67.3 (44 to 89 ). Thirty cases had low-grade urothelial carcinoma while the rest, 23, were in high-grade due to tumor grading. Tumor staging was carried out following TNM staging and the WHO classification system $[26,27]$. There were 30 cases in stage $\mathrm{Ta}+\mathrm{T} 1$ and 23 cases in stage T2 + T3. Nine of them were with LN metastasis, and 3 of them were with distant metastasis. None of the patients had been treated with any radiotherapy, chemotherapy, or vesicle instillation before surgery. The average diameter of resected tumors was $2.5 \pm 1.4 \mathrm{~cm}$. The high expression rate of claudin -4 and ZONAB is elevated, while that of ZO-1 is decreased in BC samples.

In order to identify the clinical significance of altered tight junction protein expression, the correlation between claudin-4, ZO-1, ZONAB expression, and clinicopathological factors was analyzed.

As regards claudin-4, no significant difference was found between BC cell lines (5637: $0.88 \pm 0.38$, UM-UC-3: $0.93 \pm$ 0.27 , and T24: $0.99 \pm 0.31$ ) and that of noncancer urothelial cell line sv-huc-1 $(1.0 \pm 0.29, P>0.05$, Figure 1(a)). The claudin-4 mRNA expression showed no significant change in BC cell lines (5637: $0.88 \pm 0.08$, UM-UC-3: $0.84 \pm 0.46$, and T24: $0.98 \pm 0.36)$ over that of noncancer urothelial cell line sv-huc-1 $(1.0 \pm 0.47, P>0.05$, Figure $2(\mathrm{a}))$ but was significantly upregulated in $\mathrm{BC}$ tissue $(15.50 \pm 2.76)$ over that of ANTT $(1.0 \pm 0.19, P<0.05$, Figure 2(b)). Immunohistochemical staining of the claudin- 4 protein showed strong cytoplasmic staining in ANTT and BC tissues (Figure 3(a)). The rate of high expression levels of claudin- 4 was $22.6 \%$ in ANTT (12/53) and $43.4 \%$ in BC tissues $(23 / 53, P<0.05$, Figure 3(a)).

ZO-1 protein expression was markedly decreased in $\mathrm{BC}$ cell lines (UM-UC-3: $0.63 \pm 0.22$, T24: $0.58 \pm 0.24$ ) over that of noncancer urothelial cell line sv-huc-1 $(1.0 \pm 0.33, P<$ 0.05 , Figure 1(b)). ZO-1 mRNA expression appeared to significantly decrease in BC cell lines (5637: $0.29 \pm 0.07$, UMUC-3: $0.51 \pm 0.04$, and T24: $0.21 \pm 0.05$ ) over that of noncancer urothelial cell line sv-huc-1 $(1.0 \pm 0.13, P<0.05$, Figure 2(a)) and to significantly decrease in $\mathrm{BC}$ tissue $(0.42 \pm 0.12)$ over that of ANTT $(1.0 \pm 0.19, P<0.05$, Figure 2(b)). There was strong $\mathrm{ZO}-1$ membranous staining in ANTT and weak cytoplasmic staining in BC tissues (Figure 3(b)). The rate of high expression levels of $\mathrm{ZO}-1$ was $81.1 \%$ in ANTT (43/53) and $60.4 \%$ in BC tissues (32/53, $P<0.05$, Figure 3(b)). Immunohistochemical staining showed that a high expression rate of $\mathrm{ZO}-1$ existed in 10/23 (43.5\%) muscle-invasive BC tissues, and a negative correlation between high expression rate of $\mathrm{ZO}-1$ and muscle-invasiveness was found in BC tissues (Spearman's rank order correlation coefficient: $-0.303, P<0.05$, Table 1 ).

The ZONAB protein expression was in significant upregulation in BC cell line (5637: $1.44 \pm 0.29$ ) compared to 

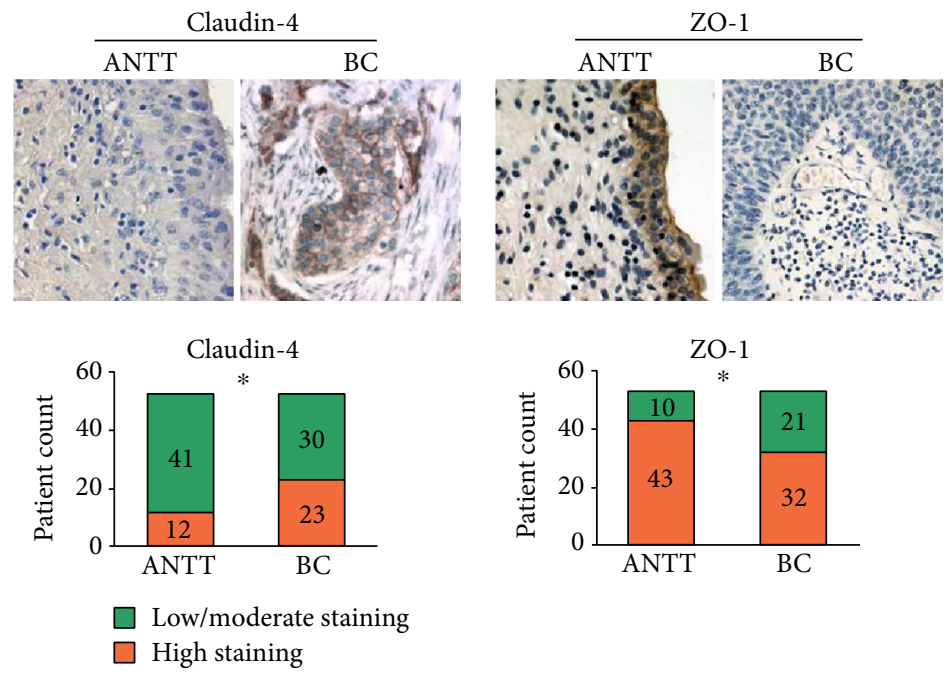

(a)

(b)
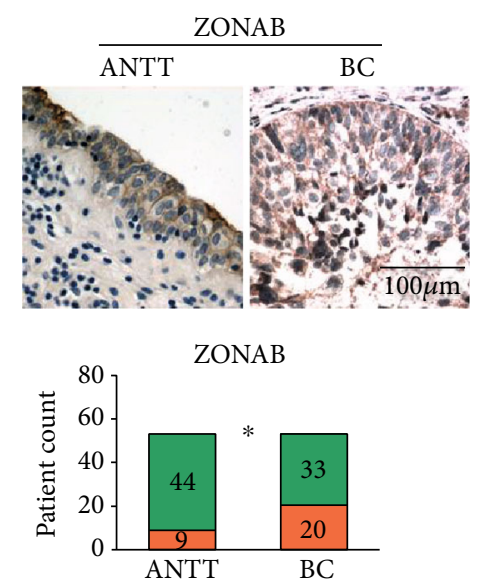

(c)

FIgure 3: The expression of tight junction proteins is altered in bladder cancer (BC) tissues. Claudin-4 (a), ZO-1 (b), and ZONAB (c) expression was detected by immunohistochemical staining in adjacent nontumor tissues (ANTT) and BC tissues (original magnification $\times 400$. Scale bar, $100 \mu \mathrm{m})$. The patient counts on high staining and low/moderate staining of tight junction protein in ANTT and BC is shown by stack bar plots $(n=53) . * P<0.05$, Fisher's exact test.

noncancer urothelial cell line sv-huc-1 $(1.0 \pm 0.41, P<0.05$, Figure $1(\mathrm{c})$ ). Its mRNA expression of $\mathrm{BC}$ cell lines appeared to significantly upregulate (5637: $10.90 \pm 1.53$, UM-UC-3: $8.66 \pm 0.65$, T24: and $4.82 \pm 1.23)$ compared to noncancer urothelial cell line sv-huc-1 $\quad(1.0 \pm 0.20, \quad P<0.05$, Figure 2(a)) and to significantly increase in BC tissue $(4.97 \pm 2.48)$ over that of ANTT $(1.0 \pm 0.48, P<0.05$, Figure 2(b)). Strong membranous staining of the ZONAB protein in ANTT and strong cytoplasmic staining in BC tissues were observed (Figure 3(c)). The rate of high expression levels of ZONAB was $17.0 \%$ in ANTT (9/53) and $37.7 \%$ in $\mathrm{BC}$ tissues $(20 / 53, P<0.05$, Figure $3(\mathrm{c}))$. ZONAB is negatively correlated to ZO- 1 as regards to the high expression rate in $\mathrm{BC}$ tissues, and they are correlated to muscle-invasive $\mathrm{BC}$ lesions. The negative correlation between the expression of ZO-1 and $\mathrm{ZONAB}$ suggests that upregulation of ZONAB is correlated with the attenuation of tight junction function in $\mathrm{BC}$, as $\mathrm{ZO}$ 1 is a critical anchoring protein for intact tight junction function. Furthermore, immunohistochemical staining of claudin-
4, ZO-1, and ZONAB showed that a positive correlation between high expression rate of ZONAB and muscleinvasiveness was observed in BC tissues (Spearman's rank order correlation coefficient: $0.418, P<0.05$, Table 1 ).

These findings suggest that the tight junction component is involved in the tumorigenesis of BC. No significant correlation between tight junction proteins and other pathological factors like gender, age, local LN, and distant metastasis was found $(P>0.05$, Table 1$)$.

\section{Discussion}

Extensive expression alteration of tight junction proteins and its tumorigenic character were observed within solid tumors $[2,28]$. We observed the change of tight junction protein expression in BC.

High expression rate of $\mathrm{ZONAB}$ is negatively correlated with $\mathrm{ZO}-1$, and high expression rate of $\mathrm{ZO}-1$ and $\mathrm{ZONAB}$ is correlated with muscle-invasiveness of BC. 
TABLE 1: Correlation between claudin-4, ZO-1, and ZONAB expressions and clinicopathological factors of bladder cancer detected by immunohistochemical test.

\begin{tabular}{|c|c|c|c|c|c|c|c|}
\hline \multirow{2}{*}{ Index } & \multirow{2}{*}{$n$} & \multicolumn{6}{|c|}{ High expression } \\
\hline & & Claudin-4 (\%) & $P$ & ZO-1 (\%) & $P$ & ZONAB (\%) & $P$ \\
\hline \multicolumn{8}{|l|}{ Gender } \\
\hline Male & 42 & $18(42.9)$ & 0.880 & $25(59.5)$ & 0.808 & $16(38.1)$ & 0.918 \\
\hline Female & 11 & $5(45.5)$ & & $7(63.6)$ & & $4(36.4)$ & \\
\hline \multicolumn{8}{|c|}{ Age (years) } \\
\hline$>65$ & 36 & $15(41.7)$ & 0.718 & $22(61.1)$ & 0.877 & $11(30.6)$ & 0.121 \\
\hline$\leq 65$ & 17 & $8(47.1)$ & & $10(58.8)$ & & $9(52.9)$ & \\
\hline \multicolumn{8}{|c|}{ Muscle invasion } \\
\hline+ & 23 & $11(47.8)$ & 0.578 & $10(43.5)$ & $0.028^{\mathrm{a}}$ & $14(60.9)$ & $0.002^{\mathrm{b}}$ \\
\hline- & 30 & $12(40.0)$ & & $22(73.3)$ & & $6(20.0)$ & \\
\hline \multicolumn{8}{|c|}{ Lymph node metastasis } \\
\hline+ & 9 & $2(22.2)$ & 0.166 & $4(44.4)$ & 0.292 & $2(22.2)$ & 0.301 \\
\hline- & 44 & $21(47.7)$ & & $28(63.6)$ & & $18(40.9)$ & \\
\hline \multicolumn{8}{|c|}{ Distant metastasis } \\
\hline+ & 3 & $1(33.3)$ & 0.412 & $2(66.7)$ & 0.823 & $1(33.3)$ & 0.874 \\
\hline- & 50 & $22(44.0)$ & & $30(60.0)$ & & $19(38.0)$ & \\
\hline
\end{tabular}

${ }^{\mathrm{a}}$ Spearman's rank order correlation coefficient: -0.303 ; there is a negative correlation $(P<0.05)$. ${ }^{\mathrm{b}}$ Spearman's rank order correlation coefficient: 0.418 ; there is a positive correlation $(P<0.05)$.

Claudin- 4 belongs to the claudin family and is encoded by CLDN4 gene. Knockdown of claudin-4 can inhibit cell motility [29]. CLDN4 gene is removed in the WilliamsBeuren syndrome, and its abnormal expression is observed in several malignancies [30]. The expression of claudin-4 is distinctly reduced in nonmelanoma skin cancer and colorectal cancer [20,31], while its expression is upregulated in several malignancies which might be used as diagnostic and therapeutic approach. Michl et al. found that cluadin-4 may be a potential therapy for cancer by acting as a specific receptor for Clostridium perfringens enterotoxin (CPE) [32]. Neesse et al. developed a novel C-CPE-Cy5.5-mediated imaging method for pancreatic tumors [33]. We observed the upregulation of claudin-4 in BC tissues. Claudin- 4 expression has prognostic value for some tumors. For example, overexpression of claudin-4 suggested shorter 5-year progression-free survival and overall survival of ovarian cancer [34], while low expression of claudin-4 indicated a shorter disease-free survival of breast carcinoma in situ [35].

$\mathrm{ZO}-1$ is encoded by TJP1 gene and serves in regulation of adherent. Altered expression of ZO-1 is found in many solid tumors. Its expression is correlated with malignant phenotypes of gastrointestinal stromal tumor [36]. ZO-1 redistribution also regulates cell dissociation in pancreatic cancer [37]. We observed that ZO-1 was downregulated both in some BC cell lines and tissues, and high ZO-1 expression was negatively correlated with muscle invasion of $\mathrm{BC}$, which means that downregulation of ZO-1 might be involved in $\mathrm{BC}$ invasion. Regular ZO-1 expression is critical for maintaining barrier function against tumor development. Disrupted ZO-1 expression promotes dissociation of pancreatic cancer cells [37]. High expression of $\mathrm{ZO}-1$ is related to better prognosis of non-small cell lung cancer [22], while decreased expres- sion of ZO-1 in colorectal cancer connects with liver metastasis closely [38].

ZONAB is encoded by the YBX3 gene, and it has doublestranded DNA-binding activity and transcription factor activity via binding to the GM-CSF promoter, as well as activity binding to intact mRNA or RNA fragments containing the $5^{\prime}$-UCCAUCA- $3{ }^{\prime}$ sequence $[39,40]$. When cell density is decreased, ZONAB is activated and transferred to the nucleus as a transcription factor [41]. Expression levels of ZONAB mRNA appeared higher in hepatocellular carcinoma (HCC) than that in corresponding nontumor tissues [42]. ZONAB expression was increased in gastric cancer which acts as a significant part in its pathogenesis and development [43]. Our result indicated that there was overexpression of ZONAB in $\mathrm{BC}$, and high ZONAB expression was positively correlated with muscle invasion of $\mathrm{BC}$, which is consistent with the results of Liu RT and his colleagues' study in colorectal cancer [44]. The tumor-promoting role of ZONAB might be caused by its activation and binding to p21 mRNA to enhance cell survival [45]. Since ZO-1 and ZONAB are critical modulators determining the switching time for epithelial cells along proximal tubules changing from a proliferative (nuclear/cytoplasmic ZONAB) state to a differentiated (ZO-1-bound ZONAB) one [46], we monitored the high expression rate of ZO-1 and ZONAB in $\mathrm{BC}$ and observed a negative correlation between their expression, which means that ZO-1 and ZONAB interaction might be involved in $\mathrm{BC}$ development.

\section{Conclusion}

In summary, the expression of tight junction proteins was significantly altered in BC. The expression of ZO-1 and 
ZONAB might be involved in muscle invasion of BC lesions and $\mathrm{ZO}-1$, and ZONAB interaction might be involved in $\mathrm{BC}$ development. Further study is required for proving the invasion-promoting character of ZO-1, for ZONAB interaction both in vitro and in vivo, and for revealing the potential signaling pathway by which these tight junction proteins regulate the invasion and metastasis of BC.

\section{Data Availability}

All data generated or analyzed during this study are included in this article.

\section{Ethical Approval}

The study was approved by the Ethics Committee of the Shengjing Hospital, China Medical University (Shenyang, Liaoning, China). All procedures carried out in this study were in consistence with the ethical standards of the Ethics Committee of the Shengjing Hospital, China Medical University (Shenyang, Liaoning, China).

\section{Conflicts of Interest}

The authors declare that they have no conflicts of interest.

\section{Acknowledgments}

The current study was supported by the China Postdoctoral Fund from China Postdoctoral Science Foundation (No. 2017M611285) and National Natural Science Foundation Youth Fund from National Natural Science Foundation of China (No. 81501292). We would like to give thanks to Mrs. Muli Zhao of China Medical University for her kind guidance on cell line culture.

\section{References}

[1] J. L. Godwin, J. Hoffman-Censits, and E. Plimack, "Recent developments in the treatment of advanced bladder cancer," Urologic Oncology, vol. 36, no. 3, pp. 109-114, 2018.

[2] T. A. Martin and W. G. Jiang, "Loss of tight junction barrier function and its role in cancer metastasis," Biochimica et Biophysica Acta, vol. 1788, no. 4, pp. 872-891, 2009.

[3] A. E. Farkas, C. T. Capaldo, and A. Nusrat, "Regulation of epithelial proliferation by tight junction proteins," Annals of the New York Academy of Sciences, vol. 1258, no. 1, pp. 115-124, 2012.

[4] F. Gao, A. Alwhaibi, S. Artham, A. Verma, and P. R. Somanath, "Endothelial Akt1 loss promotes prostate cancer metastasis via $\beta$-catenin-regulated tight-junction protein turnover," British Journal of Cancer, vol. 118, no. 11, pp. 1464-1475, 2018.

[5] K. Dokladny, M. N. Zuhl, and P. L. Moseley, "Intestinal epithelial barrier function and tight junction proteins with heat and exercise," Journal of Applied Physiology, vol. 120, no. 6, pp. 692-701, 2016.

[6] A. Luciani, B. P. Festa, Z. Chen, and O. Devuyst, "Defective autophagy degradation and abnormal tight junctionassociated signaling drive epithelial dysfunction in cystinosis," Autophagy, vol. 14, pp. 1-3, 2018.
[7] Y. Lv and L. Fu, "The potential mechanism for Hydroxysafflor yellow a attenuating blood-brain barrier dysfunction via tight junction signaling pathways excavated by an integrated serial affinity chromatography and shotgun proteomics analysis approach," Neurochemistry International, vol. 112, pp. 38-48, 2018.

[8] P. Wu, Y. Liu, W. D. Jiang et al., "Intestinal immune responses of Jian carp against Aeromonas hydrophila depressed by choline deficiency: varied change patterns of mRNA levels of cytokines, tight junction proteins and related signaling molecules among three intestinal segments," Fish \& Shellfish Immunology, vol. 65, pp. 34-41, 2017.

[9] F. J. Boivin and K. M. Schmidt-Ott, "Transcriptional mechanisms coordinating tight junction assembly during epithelial differentiation," Annals of the New York Academy of Sciences, vol. 1397, no. 1, pp. 80-99, 2017.

[10] K. Shigetomi and J. Ikenouchi, "Regulation of the epithelial barrier by post-translational modifications of tight junction membrane proteins," Journal of Biochemistry, vol. 163, no. 4, pp. 265-272, 2018.

[11] X. Chi, X. Zhao, W. Wang et al., "Fusion expression of Occludin extracellular loops and an $\alpha$-helical bundle: a new research model for tight junction," PLoS One, vol. 12, no. 4, article e0175516, 2017.

[12] A. Piontek, J. Rossa, J. Protze et al., "Polar and charged extracellular residues conserved among barrier-forming claudins contribute to tight junction strand formation," Annals of the New York Academy of Sciences, vol. 1397, no. 1, pp. 143-156, 2017.

[13] L. S. Rodgers, M. T. Beam, J. M. Anderson, and A. S. Fanning, "Epithelial barrier assembly requires coordinated activity of multiple domains of the tight junction protein ZO-1," Journal of Cell Science, vol. 126, no. 7, pp. 1565-1575, 2013.

[14] K. Chishiki, S. Kamakura, J. Hayase, and H. Sumimoto, "Ric$8 \mathrm{~A}$, an activator protein of G $\alpha \mathrm{i}$, controls mammalian epithelial cell polarity for tight junction assembly and cystogenesis," Genes to Cells, vol. 22, no. 3, pp. 293-309, 2017.

[15] A. Buckley and J. R. Turner, "Cell biology of tight junction barrier regulation and mucosal disease," Cold Spring Harbor Perspectives in Biology, vol. 10, no. 1, 2018.

[16] T. A. Martin, R. E. Mansel, and W. G. Jiang, "Loss of occludin leads to the progression of human breast cancer," International Journal of Molecular Medicine, vol. 26, no. 5, pp. 723734, 2010.

[17] L. Zhang, T. Feng, and L. J. Spicer, "The role of tight junction proteins in ovarian follicular development and ovarian cancer," Reproduction, vol. 155, no. 4, pp. R183-R198, 2018.

[18] W. Jia, T. A. Martin, G. Zhang, and W. G. Jiang, "Junctional adhesion molecules in cerebral endothelial tight junction and brain metastasis," Anticancer Research, vol. 33, no. 6, pp. 2353-2359, 2013.

[19] M. A. Abd-Elazeem and M. A. Abd-Elazeem, "Claudin 4 expression in triple-negative breast cancer: correlation with androgen receptors and Ki-67 expression," Annals of Diagnostic Pathology, vol. 19, no. 1, pp. 37-42, 2015.

[20] V. Hahn-Stromberg, S. Askari, A. Ahmad, R. Befekadu, and T. K. Nilsson, "Expression of claudin 1, claudin 4, and claudin 7 in colorectal cancer and its relation with CLDN DNA methylation patterns," Tumour Biology, vol. 39, no. 4, p. 101042831769756, 2017.

[21] O. Ondic, H. Sidlova, and R. Alaghehbandan, "Claudin-4 expression is associated with survival in ovarian cancer but 
not with chemotherapy response," International Journal of Gynecological Pathology, vol. 39, 2020.

[22] S. Ni, L. Xu, J. Huang et al., "Increased ZO-1 expression predicts valuable prognosis in non-small cell lung cancer," International Journal of Clinical and Experimental Pathology, vol. 6, no. 12, pp. 2887-2895, 2013.

[23] M. Liu, J. Yang, Y. Zhang et al., "ZIP4 promotes pancreatic cancer progression by repressing ZO-1 and claudin-1 through a ZEB1-dependent transcriptional mechanism," Clinical Cancer Research, vol. 24, no. 13, pp. 3186-3196, 2018.

[24] J. E. Jimenez-Salazar, P. Posadas-Rodriguez, R. C. LazzariniLechuga et al., "Membrane-initiated estradiol signaling of epithelial-mesenchymal transition-associated mechanisms through regulation of tight junctions in human breast cancer cells," Hormones and Cancer, vol. 5, no. 3, pp. 161173, 2014.

[25] H. K. Na and J. Y. Lee, "Molecular basis of alcohol-related gastric and colon cancer," International Journal of Molecular Sciences, vol. 18, no. 6, p. 1116, 2017.

[26] P. A. Humphrey, H. Moch, A. L. Cubilla, T. M. Ulbright, and V. E. Reuter, "The 2016 WHO classification of tumours of the urinary system and male genital organs-part B: prostate and bladder tumours," European Urology, vol. 70, no. 1, pp. 106-119, 2016.

[27] M. Babjuk, A. Böhle, M. Burger et al., "EAU Guidelines on non-muscle-invasive urothelial carcinoma of the bladder: update 2016," European Urology, vol. 71, no. 3, pp. 447-461, 2017.

[28] A. O. Leech, R. G. Cruz, A. D. Hill, and A. M. Hopkins, "Paradigms lost-an emerging role for over-expression of tight junction adhesion proteins in cancer pathogenesis," Annals of Translational Medicine, vol. 3, no. 13, p. 184, 2015.

[29] P. G. Webb, M. A. Spillman, and H. K. Baumgartner, "Claudins play a role in normal and tumor cell motility," BMC Cell Biology, vol. 14, no. 1, p. 19, 2013.

[30] A. L. Lameris, S. Huybers, J. R. Burke, L. A. Monnens, R. J. Bindels, and J. G. Hoenderop, "Involvement of claudin 3 and claudin 4 in idiopathic infantile hypercalcaemia: a novel hypothesis?," Nephrology, Dialysis, Transplantation, vol. 25, no. 11, pp. 3504-3509, 2010.

[31] C. Zoschke, M. Ulrich, M. Sochorova et al., "The barrier function of organotypic non-melanoma skin cancer models," Journal of Controlled Release, vol. 233, pp. 10-18, 2016.

[32] P. Michl, M. Buchholz, M. Rolke et al., "Claudin-4: a new target for pancreatic cancer treatment using Clostridium perfringens enterotoxin," Gastroenterology, vol. 121, no. 3, pp. 678684, 2001.

[33] A. Neesse, A. Hahnenkamp, H. Griesmann et al., "Claudin-4targeted optical imaging detects pancreatic cancer and its precursor lesions," Gut, vol. 62, no. 7, pp. 1034-1043, 2013.

[34] L. Martin de la Fuente, S. Malander, L. Hartman et al., "Claudin-4 expression is associated with survival in ovarian cancer but not with chemotherapy response," International Journal of Gynecological Pathology, vol. 37, no. 2, pp. 101-109, 2018.

[35] G. M. Duarte, N. R. Almeida, F. Tocchet et al., "Claudin-4 expression is associated with disease-free survival in breast carcinoma-in-situ: mean follow-up of 8.2 years," Clinical Breast Cancer, vol. 18, no. 5, pp. e1111-e1116, 2018.

[36] H. Zhu, J. Lu, X. Wang et al., "Upregulated ZO-1 correlates with favorable survival of gastrointestinal stromal tumor," Medical Oncology, vol. 30, no. 3, p. 631, 2013.
[37] X. Tan, H. Egami, S. Ishikawa, T. Kurizaki, M. Hirota, and M. Ogawa, "Zonula occludens-1 (ZO-1) redistribution is involved in the regulation of cell dissociation in pancreatic cancer cells," Digestive Diseases and Sciences, vol. 50, no. 8, pp. 1402-1409, 2005.

[38] T. Kaihara, T. Kusaka, M. Nishi et al., "Dedifferentiation and decreased expression of adhesion molecules, E-cadherin and $\mathrm{ZO}-1$, in colorectal cancer are closely related to liver metastasis," Journal of Experimental \& Clinical Cancer Research, vol. 22, no. 1, pp. 117-123, 2003.

[39] L. S. Coles, P. Diamond, F. Occhiodoro, M. A. Vadas, and M. F. Shannon, "Cold shock domain proteins repress transcription from the GM-CSF promoter," Nucleic Acids Research, vol. 24, no. 12, pp. 2311-2317, 1996.

[40] R. Petruzzelli, S. Gaudino, G. Amendola et al., "Role of the cold shock domain protein $\mathrm{A}$ in the transcriptional regulation of HBG expression," British Journal of Haematology, vol. 150, no. 6, pp. 689-699, 2010.

[41] P. Frankel, A. Aronheim, E. Kavanagh et al., "RalA interacts with ZONAB in a cell density-dependent manner and regulates its transcriptional activity," The EMBO Journal, vol. 24, no. 1, pp. 54-62, 2004.

[42] M. Yasen, G. Obulhasim, K. Kajino et al., "DNA binding protein a expression and methylation status in hepatocellular carcinoma and the adjacent tissue," International Journal of Oncology, vol. 40, no. 3, pp. 789-797, 2011.

[43] G. R. Wang, Y. Zheng, X. M. Che et al., "Upregulation of human DNA binding protein A (dbpA) in gastric cancer cells," Acta Pharmacologica Sinica, vol. 30, no. 10, pp. 1436-1442, 2009.

[44] R. T. Liu, G. R. Wang, C. Liu et al., "RNAi-mediated downregulation of DNA binding protein A inhibits tumorigenesis in colorectal cancer," International Journal of Molecular Medicine, vol. 38, no. 3, pp. 703-712, 2016.

[45] M. Nie, M. S. Balda, and K. Matter, "Stress- and rho-activated $\mathrm{ZO}-1$-associated nucleic acid binding protein binding to $\mathrm{p} 21$ mRNA mediates stabilization, translation, and cell survival," Proceedings of the National Academy of Sciences of the United States of America, vol. 109, no. 27, pp. 10897-10902, 2012.

[46] A. Pozzi and R. Zent, "ZO-1 and ZONAB interact to regulate proximal tubular cell differentiation," Journal of the American Society of Nephrology, vol. 21, no. 3, pp. 388-390, 2010. 